Produto \& Produção, vol.17 n.3, p.1-14, out. 2016

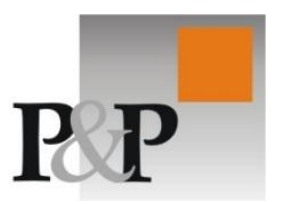

RECEBIDO EM 19/09/2016. ACEITO EM 26/09/2016.

\title{
Análise das Ferramentas de TIC que contribuem na Integração Cliente-Fornecedor para Desenvolvimento de Produto
}

\author{
Daisy Valle Enrique \\ Universidade Federal do Rio Grande do Sul - UFRGS \\ daisy.enrique@ufrgs.br
}

Bernardo de Abreu Bortoluzzi Souza

Universidade Federal do Rio Grande do Sul - UFRGS

bernardo.bortoluzzi@ufrgs.br

\author{
Néstor Fabián Ayala \\ Universidade Federal do Rio Grande do Sul - UFRGS \\ ayala@producao.ufrgs.br
}

\author{
Giuliano Almeida Marodin \\ University of South Carolina - USC \\ gmarodin@moore.sc.edu
}

\section{RESUMO}

Práticas colaborativas entre clientes e fornecedores são essenciais para o desenvolvimento de produtos de qualidade. Esta pesquisa examina o uso das ferramentas de tecnologia da informação e comunicação (TIC) no processo de desenvolvimento de produto (PDP) colaborativo, assim como o impacto que a colaboração pode trazer ao desempenho das empresas. Para tanto, foi realizado uma pesquisa survey em 240 empresas da região Sul do Brasil, seguida de análises de dados, através de regressão lineal (OLS), e que por fim, apontaram relação positiva entre o uso das tecnologias, através de práticas colaborativas, com a satisfação dos clientes e tempo de entrega dos produtos.

Palavras-chave: desenvolvimento de produto, colaboração, tecnologia da informação e comunicação.

\begin{abstract}
Collaborative practices among customers and suppliers are essential to the development of quality products. This research examines the use of information and communication technology tools (ICT) in the collaborative new product development process (NPD), as well as the impact that collaboration can bring the performance of the companies. Thus, was applied a survey research in 240 companies in
\end{abstract}


the South region of Brazil, followed by data analysis using linear regression (OLS), and finally showed a positive relationship among the use of technology through collaborative practices with satisfaction customer and delivery time of the products.

Key Words: new product development, collaboration, information and communication technologies

\section{Introdução}

Com a concorrência global, o mercado tornou-se mais competitivo, exigindo das empresas uma busca incessante pela vantagem competitiva, que segundo Porter (1989) ocorre quando uma empresa consegue se distinguir dos seus concorrentes ao apresentar um valor superior para seus clientes. Assim, diversos fatores são utilizados pelas empresas no intuito de possibilitar um desempenho superior em relação aos concorrentes do setor. Um destes fatores, que nos últimos tempos tem sido cada vez mais utilizado, é o de cooperação cliente-fornecedor, na qual vem colhendo frutos na busca pela vantagem competitiva.

Esta relação cliente-fornecedor, que antes era vista basicamente como uma disputa entre partes através das negociações de preço, agora se vê em forma de cooperação, que se torna necessária para a obtenção de vantagem competitiva no mercado. Desta forma, a aproximação pode contribuir para o desenvolvimento de produtos e compartilhamento de informações e tecnologias.

A participação do fornecedor no processo de desenvolvimento de produtos é uma atividade que vem se tornando mais frequente em diversas indústrias. No entanto, esta é uma questão que divide os pesquisadores em relação a sua contribuição para a empresa. Por um lado, estão os que acreditam que o processo torna-se mais rápido e produtivo (FETTERMANN, 2008), reduz custos e diminui a chance de atrasos no projeto (BORNIA \& LORANDI, 2008). Do outro, estão os que questionam essa prática, descrevendo uma série de riscos, como no caso da exposição da empresa e diminuição da flexibilidade do comprador (CAMPOS, 2012).

O desafio que deve ser tomado é o de gerenciar a integração das diversas áreas das empresas, principalmente aquelas que têm maior relação com o PDP, este gerenciamento será um fator importantíssimo em busca da minimização destes riscos (SANTOS et al.,2010). Em busca desta integração, as empresas devem levar em conta a facilidade no compartilhamento de informações, que às vezes, principalmente com o distanciamento geográfico, torna-se complicado. Para isso, têm-se como alternativas as ferramentas de tecnologia da informação e comunicação (TIC). Tais ferramentas de TIC têm por objetivo fazer com que a comunicação entre as empresas seja mais eficiente e colaborem para o processo de desenvolvimento de produtos.

Portanto, o objetivo deste artigo é examinar e entender a utilização de ferramentas de TIC no PDP colaborativo e seu consequente impacto na performance da empresa. Para tanto, este trabalho propõe uma pesquisa qualitativa que busca explorar, as ferramentas de TIC utilizadas pelas empresas do Sul do Brasil, assim como de que forma essas ferramentas auxiliam na prática colaborativa entre cliente-fornecedor e também como elas beneficiam as empresas que as utilizam. Desta forma o trabalho servirá de orientação para as empresas que buscam esta integração em prol da melhoria dos processos e produtos.

\section{Referencial teórico}

\subsection{Processo de desenvolvimento de produto (PDP)}

O Processo de Desenvolvimento de Produto (PDP) consiste no conjunto de atividades que se iniciam com a análise das necessidades de mercado, das possibilidades e restrições tecnológicas, dos recursos disponíveis, e que devem estar alinhadas as estratégias competitivas e de produtos da empresa. Este processo procura chegar às especificações de projeto de um produto e de seu processo de produção, além de acompanhar o produto no mercado, após o lançamento, para identificar eventuais mudanças necessárias, delinear a descontinuidade do produto no mercado e adicionar, no PDP, as lições aprendidas ao longo do ciclo de vida do produto (ROZENFELD et al., 2006). 
No mercado atual, onde a concorrência é grande, é cada vez maior a importância que as empresas têm dado ao PDP. A capacidade de a empresa introduzir novos produtos no mercado com conteúdo tecnológico e características de qualidade, custo, agilidade e produtividade que atendam as exigências dos consumidores é uma das formas de a empresa obter vantagem competitiva no mercado (FARIA et al., 2008).

Um PDP eficiente e eficaz, por sua vez pode, além de obter vantagem competitiva para a empresa, trazer benefícios internos para a organização que até mesmo extrapolam as barreiras da empresa e afetam a cadeia de suprimentos. Entre estes benefícios, podem ser citados desde ganhos de flexibilidade até uma abertura para exploração de novos mercados (SANTOS et al., 2010).

Contudo, o desenvolvimento de produtos é um processo complexo e que exige a integração dos diversos setores da empresa. Habilidades e informações advindas de membros de diversas áreas são necessárias (MUNDIN et al., 2002). Entretanto, esta integração deve considerar não somente as áreas internas da empresa, mas também os agentes externos, como os fornecedores, capazes de melhorar o desempenho do PDP da empresa.

\subsection{Participação do fornecedor no PDP}

A interação cliente-fornecedor já ocorre normalmente nos processos de prestação de serviços, suprimento de mercadorias e fluxo de informações e finanças (SANTOS et al., 2010). O que é mais recente nesta relação é a integração no âmbito do PDP, que para se ter êxito exige relacionamentos mais estreitos entre as duas partes. Segundo Handfield et al. (1999), o envolvimento precoce do fornecedor (Early Suppliers Involvment - ESI) no processo de desenvolvimento e design de produtos está associado a benefícios em diversas dimensões da empresa, incluindo qualidade, custo e tempo de ciclo do produto.

Apesar dos benefícios, existem alguns obstáculos que impedem o andamento da integração cliente-fornecedor e que devem ser superados para que este processo tenha sucesso. Conforme Ragatz et al. (1997), as principais barreiras existentes são a de resistência de tanto o cliente como o fornecedor em compartilhar informações próprias um com o outro e também a disputa na autoria das invenções, que pode incentivar designers e engenheiros das empresas a não desistir do controle sobre as decisões de projeto.

Ragatz et al. (1997) também traz algumas formas de superar ou minimizar estas barreiras e que, segundo ele, se dividem em dois blocos: estrutura do relacionamento e alocação de recursos, como apresentado na Figura 1. O primeiro bloco, denominado estrutura do relacionamento, expressa formas de facilitar a integração ajudando a quebrar barreiras internas e externas. A estrutura é caracterizada por: compromisso da alta gerência de ambas as empresas, compartilhamento de iniciativas para educação e treinamento, entre outras. $\mathrm{O}$ segundo bloco, denominado de alocação de recursos, afeta diretamente a integração cliente-fornecedor no PDP. Os recursos são de ordem intelectual, humana e física.

Neste sentido, Lawson et al. (2009) afirma que o compartilhamento de conhecimento e mecanismos de socialização são dois pontos chaves para o sucesso deste processo, e que por sua vez podem superar as barreiras comportamentais e culturais existentes nesta relação cliente-fornecedor. 


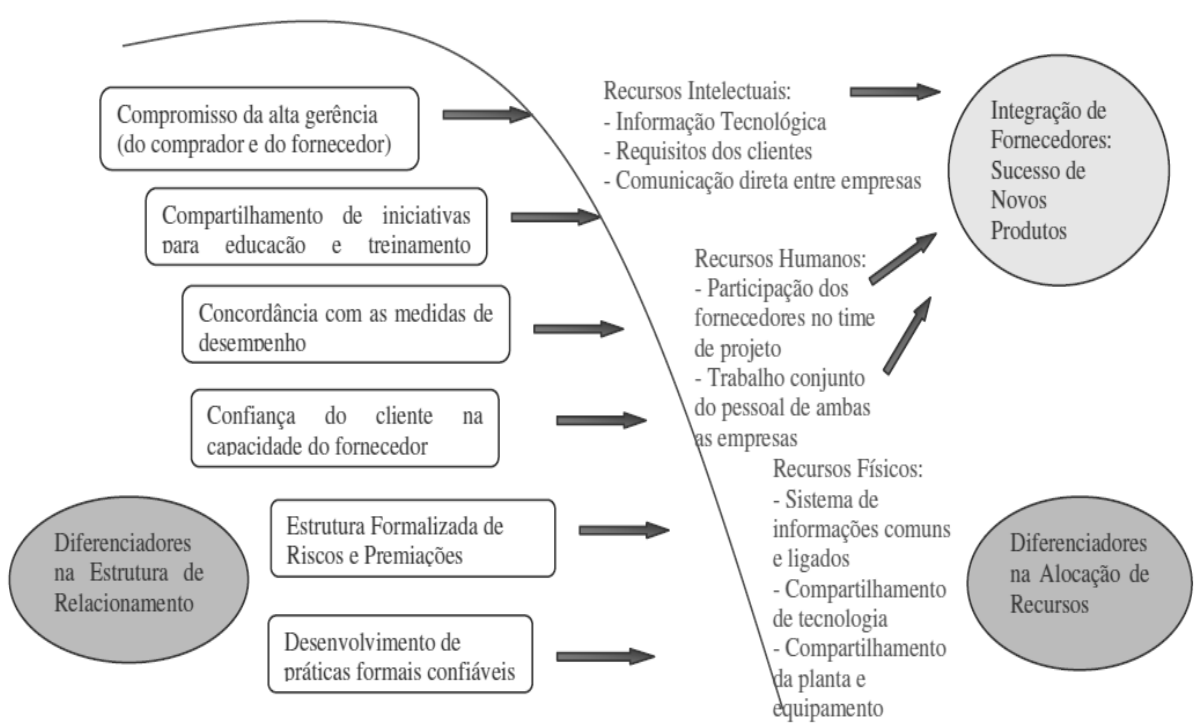

Figura 1. Fatores críticos que afetam a integração de fornecedores no PDP Fonte: Ragatz et al. (1997)

\subsection{Compartilhamento de conhecimento através da configuração de envolvimento do fornecedor}

Uma forma de classificar o compartilhamento de conhecimento entre Cliente-Fornecedor é o do grau de envolvimento do fornecedor no PDP. Desta forma, assim como ilustrado na Figura 2, têmse geralmente as seguintes classificações: (i) $\mathrm{O}$ fornecedor se envolve nas fases preliminares do PDP para apoiar nas diretrizes do projeto (Caixa branca - White Box); (ii) Trabalho conjunto do cliente e fornecedor para desenvolvimento do produto (Caixa Cinza - Grey Box); e (iii) projeto de responsabilidade total do fornecedor a partir de especificações dadas pelo cliente (Caixa Preta - Black Box) (PETERSEN et al., 2005).

Portanto, em um extremo encontra-se a classificação white box onde o cliente coordena as decisões e especificações do projeto e o envolvimento do fornecedor está basicamente limitado ao estágio final do projeto, que consiste nas atividades industriais e de manufatura. No outro extremo está o nível black box onde o fornecedor é quem está encarregado do PDP e o cliente, por sua vez, enxerga apenas um produto pronto recebido do fornecedor de acordo com suas especificações (LE DAIN \& MERMINOD, 2014). 


\begin{tabular}{|l|l|l|}
\hline \multicolumn{1}{|c|}{ Black Box } & \multicolumn{1}{c|}{ Grey Box } & \multicolumn{1}{c|}{ White Box } \\
\hline Projeto é conduzido & Desenvolvimento e & Discussões são \\
principalmente pelo ltomada de decisão & Ifeitas sobre as \\
fornecedor, & conjunta sobre as & especificações do \\
baseado nas & especificações do & projeto mas o \\
especificações do & projeto & Icliente faz todas as \\
cliente & & decisões finais \\
\hline
\end{tabular}

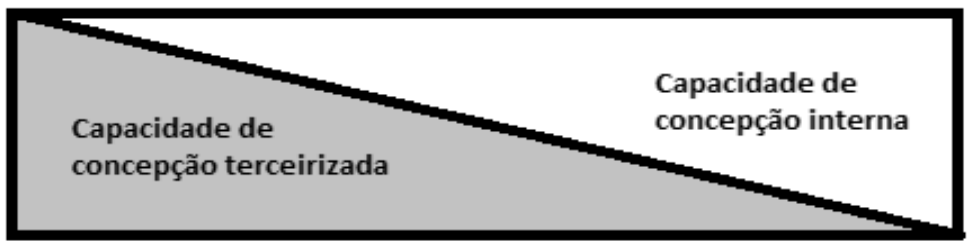

Figura 2. Espectro de envolvimento do fornecedor Fonte: Adaptado de Petersen et al. (2005).

Considerando este contexto, o PDP torna cada vez mais uma questão de colaboração entre a equipe interna de desenvolvimento da empresa central composto por pessoas de diferentes áreas (como marketing, design, engenharia, manufatura, qualidade, compras, etc.) e membros externos de fornecedores co-localizados ou distantes geograficamente (GASSMANN, 2003).

\subsection{Tecnologias da informação na gestão da cadeia de suprimentos}

No ambiente competitivo atual, as empresas buscam novas estratégias para sobreviver no mercado. Uma delas é a de não atuar mais isoladamente e sim, como membros de uma cadeia em rede. A Cadeia de Suprimentos (Supply Chain - SC), como é chamada, representa uma rede de organizações, através de ligações nos dois sentidos, dos diferentes processos e atividades que produzem valor na forma de produtos e serviços que são colocados nas mãos do consumidor final (CHRISTHOFER, 2001). Desta forma, a gestão da cadeia de suprimentos (Supply Chain Management - SCM) consiste em gerenciar a integração de todas as atividades da cadeia através da melhoria nas relações entre as empresas com o objetivo de aprimorar o desempenho da organização individualmente e da cadeia como um todo (FERRARI \& POLACISKI, 2011).

Todavia, a troca de conhecimento entre times já desenvolvidos não ocorre automaticamente. Para obter sucesso na integração da cadeia de suprimentos, são necessários processos que facilitem o uso e a transferência de conhecimento através das fronteiras funcionais e organizacionais que, às vezes, principalmente com o distanciamento geográfico, tornam-se complicados. Para isso, têm se como alternativas as ferramentas de TICs.

As TICs vêm se desenvolvendo em ritmo acelerado em todos os setores. Hoje é quase impossível trabalhar com SCM sem um auxílio de um software de computador. Estes sistemas vêm desempenhando um papel importante por sua capacidade de apoiar um aumento do nível de comunicação entre os elos.

Segundo Maçada et al. (2007), níveis de inventário inadequado, ordens de entrega e recebimento não cumpridas e problemas na transmissão de informações são os principais problemas enfrentados pelas cadeias de suprimentos e que, com o auxílio das tecnologias de informação, podem ser minimizados ou eliminados. O autor também afirma que existem diversas TICs que podem auxiliar no processamento de um maior número de informações, de maneira mais precisa, com maior frequência e de uma quantidade maior de fontes dispersas geograficamente. 
O Quadro 1 apresenta os principais tipos de tecnologias aplicadas à cadeia de suprimentos assim como suas descrições.

\section{Quadro 1. Sistemas de TIC aplicados a Cadeia de Suprimentos}

Fonte: Adaptado de Bandeira e Maçada (2007)

\begin{tabular}{|c|c|c|}
\hline Tecnologia & Descrição & Autores \\
\hline Código de Barras & $\begin{array}{c}\text { Tecnologia de alocação de códigos legiveis por computador em itens, caixas e } \\
\text { contêineres, sendo empregada para melhorar a precisão da informação e } \\
\text { velocidade de transmissão de dados. }\end{array}$ & $\begin{array}{l}\text { (BANDEIRA \& MAÇADA, 2007) } \\
\text { (ABREU, M. C., 2006) }\end{array}$ \\
\hline $\begin{array}{c}\text { Business } \\
\text { Inteligence (BI) }\end{array}$ & $\begin{array}{l}\text { Conjunto de aplicações que organiza e estrutura os dados de transação de uma } \\
\text { organização, facilitando a análise de modo a beneficiar as operaçốes e o suporte } \\
\text { às suas decisôes. }\end{array}$ & $\begin{array}{l}\text { (BANDEIRA \& MAÇADA, 2007) } \\
\text { (ABREU, M. C., 2006) } \\
\text { (HANNULA \& PIRTTIMAKI, 2003) }\end{array}$ \\
\hline $\begin{array}{l}\text { Eletronic Data } \\
\text { Interchange } \\
\text { (EDI) }\end{array}$ & $\begin{array}{c}\text { É a transferência eletrônica de dados. Divide-se em duas categorias: o EDI } \\
\text { tradicional, que utiliza serviços da VAN (rede de valor agregado), e a WebEDI, cujo } \\
\text { acesso é pela internet. }\end{array}$ & $\begin{array}{l}\text { (BANDEIRA \& MAÇADA, 2007) } \\
\text { (ABREU, M. C., 2006) } \\
\text { (MATTOS \& LAURINDO, 2015) }\end{array}$ \\
\hline $\begin{array}{l}\text { Rastreamento } \\
\text { de Frotas }\end{array}$ & $\begin{array}{l}\text { Baseada em transmissão via satélite ou pela telefonia celular para rastreamento e } \\
\text { monitoramento de veículos. Os dados gerados por esse sistema alimentam o TMS } \\
\text { e WMS. }\end{array}$ & $\begin{array}{c}\text { (BANDEIRA \& MAÇADA, 2007) } \\
\text { (FERRARI \& POLACISKI, 2011) }\end{array}$ \\
\hline $\begin{array}{c}\text { Autmated } \\
\text { Quality Control }\end{array}$ & $\begin{array}{c}\begin{array}{c}\text { Responsável pelo monitoramento dos processos de garantia da qualidade, } \\
\text { procedimentos de inspeção, especificaçốes e calibração dos instrumentos de } \\
\text { medição. }\end{array} \\
\end{array}$ & $\begin{array}{l}\text { (BANDEIRA \& MAÇADA, 2007) } \\
\text { (ABREU, M. C., 2006) }\end{array}$ \\
\hline $\begin{array}{l}\text { Manufacturing } \\
\text { Execution } \\
\text { System (MES) }\end{array}$ & $\begin{array}{l}\text { Sistemas de planejamento de utilização de recursos. Suporta o intercâmbio de } \\
\text { informações entre o planejamento da produção e o controle do processo de } \\
\text { produção através do monitoramento e acompanhamento da matéria-prima, } \\
\text { equipamento pessoal, instruções e instalações de produção. }\end{array}$ & $\begin{array}{l}\text { (BANDEIRA \& MAÇADA, 2007) } \\
\text { (FERRARI \& POLACISKI, 2011) }\end{array}$ \\
\hline $\begin{array}{c}\text { Transportation } \\
\text { Management } \\
\text { System (TMS) }\end{array}$ & $\begin{array}{l}\text { Responsável pelo controle do transporte de cargas, determinando o modal, } \\
\text { gerenciando a consolidação de fretes e coordenando os esforços de transporte. }\end{array}$ & (BANDEIRA \& MAÇADA, 2007) \\
\hline $\begin{array}{l}\text { Product Data } \\
\text { Management } \\
\text { (PDM) }\end{array}$ & $\begin{array}{l}\text { Gerencia as informaçôes relacionadas aos produtos, também servindo como uma } \\
\text { ferramenta de integração, conectando diferentes áreas de desenvolvimento de } \\
\text { produtos. }\end{array}$ & $\begin{array}{l}\text { (BANDEIRA \& MAÇADA, 2007) } \\
\text { (ABREU, M. C., 2006) }\end{array}$ \\
\hline $\begin{array}{l}\text { Supply Chain } \\
\text { Planning (SCP) }\end{array}$ & $\begin{array}{l}\text { Auxilia no planejamento, execução e mensuração dos processos, incluindo } \\
\text { módulos de previsão de demanda, planejamento de inventário e distribuição. }\end{array}$ & $\begin{array}{l}\text { (BANDEIRA \& MAÇADA, 2007) } \\
\text { (ABREU, M. C., 2006) }\end{array}$ \\
\hline $\begin{array}{c}\text { Warehouse } \\
\text { Management } \\
\text { System (WMS) }\end{array}$ & $\begin{array}{l}\text { Otimiza atividades do processo de armazenagem, rastreando e controlando o } \\
\text { movimento do inventário no depósito. Sua utilização se restringe a decisões } \\
\text { operacionais. }\end{array}$ & $\begin{array}{l}\text { (BANDEIRA \& MAÇADA, 2007) } \\
\text { (ABREU, M. C., 2006) }\end{array}$ \\
\hline $\begin{array}{l}\text { Customer } \\
\text { Relationship } \\
\text { Management } \\
\text { (CRM) }\end{array}$ & $\begin{array}{l}\text { Unifica as informaçôes sobre os clientes, centralizando as interaçôes e } \\
\text { antecipando as necessidades dos clientes. Proporciona o controle de atividades } \\
\text { promocionais e seus impactos na demanda, assim como o controle de atividades } \\
\text { de garantia de produtos. }\end{array}$ & $\begin{array}{l}\text { (BANDEIRA \& MAÇADA, 2007) } \\
\text { (ABREU, M. C., 2006) } \\
\text { (CHOY et al.,2003) }\end{array}$ \\
\hline $\begin{array}{l}\text { Radio Frequency } \\
\text { (RF) }\end{array}$ & $\begin{array}{l}\text { Facilita a comunicação, disponibilizando informações sobre os produtos. } \\
\text { Ferramenta de suporte que automatiza processos e melhora a gestão das } \\
\text { operações, eliminando falhas humanas. }\end{array}$ & $\begin{array}{l}\text { (BANDEIRA \& MAÇADA, 2007) } \\
\text { (ABREU, M. C., 2006) } \\
\text { (MATTOS \& LAURINDO, 2015) }\end{array}$ \\
\hline $\begin{array}{l}\text { Demand } \\
\text { Forecasting } \\
\text { System (DFS) } \\
\end{array}$ & $\begin{array}{l}\text { Utiliza métodos matemáticos que manipulam dados históricos e dados externos } \\
\text { para previsão de demanda por produtos e serviços. Em geral, integra outros } \\
\text { sistemas, tais como ERP e SCP. }\end{array}$ & $\begin{array}{l}\text { (BANDEIRA \& MAÇADA, 2007) } \\
\text { (ABREU, M. C., 2006) }\end{array}$ \\
\hline $\begin{array}{l}\text { Web-based } \\
\text { Information } \\
\text { System (WIS) } \\
\end{array}$ & $\begin{array}{l}\text { Facilitam os processos internos e externos das empresas, integrando sistemas } \\
\text { empresariais de informação. Na SCM, os WIS mais presentes são o e-procurement } \\
\text { e o e-market place. }\end{array}$ & $\begin{array}{l}\text { (BANDEIRA \& MAÇADA, 2007) } \\
\text { (ABREU, M. C., 2006) }\end{array}$ \\
\hline$B 2 B$ & $\begin{array}{l}\text { Mercados eletrônicos onde fornecedores e compradores interagem para conduzir } \\
\text { transações. }\end{array}$ & $\begin{array}{l}\text { (BANDEIRA \& MAÇADA, 2007) } \\
\text { (MATTOS \& LAURINDO, 2015) }\end{array}$ \\
\hline $\begin{array}{l}\text { Enterprise } \\
\text { Resource } \\
\text { Planing (ERP) }\end{array}$ & $\begin{array}{l}\text { Sistema unificado de informação que integra os departamentos e funções da } \\
\text { empresa. Melhoram o fluxo de informações da cadeia em tal grau que se tornaram } \\
\text { um padrão de operação. }\end{array}$ & $\begin{array}{l}\text { (BANDEIRA \& MAÇADA, 2007) } \\
\text { (ABREU, M. C., 2006) (KUMAR et } \\
\text { al., 2000) (ECKARTZ et al., 2009) }\end{array}$ \\
\hline $\begin{array}{c}\text { Computer Aided } \\
\text { Design (CAD) }\end{array}$ & $\begin{array}{l}\text { Permite a realização de desenhos industriais na tela do computador que podem } \\
\text { ser armazenados, manipulados e atualizados eletronicamente. }\end{array}$ & $\begin{array}{l}\text { (BANDEIRA \& MAÇADA, 2007) } \\
\text { (LICHTENBERG, 2007) (ÁLVARES \& } \\
\text { FERREIRA, 2006) }\end{array}$ \\
\hline $\begin{array}{l}\text { Sistema de } \\
\text { gestão do } \\
\text { relacionamento } \\
\text { com } \\
\text { fornecedores } \\
\text { (SRM) }\end{array}$ & $\begin{array}{l}\text { Softwares que criam um quadro comum de referência para permitir uma } \\
\text { comunicação eficaz com seus fornecedores, aumentando a eficiência dos } \\
\text { processos associados à aquisição de bens e serviços, gestão de inventário e } \\
\text { materiais de processamento. }\end{array}$ & $\begin{array}{c}\text { (LEE et al., 2007) (CHOY et } \\
\text { al.,2003) }\end{array}$ \\
\hline
\end{tabular}


Uma forma de utilização das TICs, que já é mais difundida no exterior e aos poucos ganha terreno no Brasil, é no desenvolvimento de produto colaborativo. Hoje em dia, para desenvolver produtos, as empresas se relacionam com equipes internas e externas, e as ferramentas de TIC são utilizadas para facilitar este processo.

Dentre as tecnologias que são utilizadas no SCM serão utilizadas cinco, entre as mais presentes nas empresas, neste trabalho: ERP, EDI, BI, SRM e CAD. Assim, propõe-se a seguinte hipótese com seus desdobramentos:

H1: O uso de tecnologias de informação, tais como ERP (Hla), EDI (H1b), BI (H1c), SRM (Hld), e CAD (H1e), contribui para o aumento da colaboração entre a empresa e seus fornecedores para o desenvolvimento de produtos.

\subsection{Impacto do PDP colaborativo na performance da empresa}

A adoção de TIC está cada vez mais sendo buscado pelas empresas, incluindo o uso da TIC para o PDP com o intuito de melhorar produtividade, colaboração, comunicação, gestão do conhecimento, qualidade do produto, tempo de entrega, desempenho de mercado, entre outros (Ozer, 2000; Barczak et al., 2007). De acordo com Barczak et al. (2007), o impacto da utilização de ferramentas de TIC no PDP consiste em duas medidas: performance de mercado e velocidade até o mercado.

As TICs facilitam a colaboração intra/inter-organizacional, que é essencial para o desenvolvimento de produto colaborativo, na medida em que fornece plataformas de comunicação, transferência de conhecimento e trabalho sincronizado. (Silva, 2013)

No modelo apresentado, em relação à performance do produto, a satisfação dos clientes e o tempo de entrega serão as dimensões utilizadas. A Satisfação do Cliente é definida como avaliação do cliente, sobre tudo, com a performance do que esta sendo oferecido (Johnson and Fornell, 1991). Nessa perspectiva, a satisfação do cliente é delineada como a avaliação feita pelos clientes em relação aos produtos e serviços que alcancem as suas expectativas (Yi, 1991). Assim, é proposta a seguinte hipótese:

H3: A adoção de práticas colaborativas está positivamente associada com o aumento da satisfação dos clientes.

O tempo de entrega do produto está relacionado com o tempo que o produto leva desde a concepção da idéia até a sua entrega para o cliente. (Barczak et al., 2007). Quanto menor for este processo, mais rápido o produto é entregue ao cliente. Assim, é proposta a seguinte hipótese:

H4: A adoção de práticas colaborativas está positivamente associada com a diminuição do tempo de entrega dos produtos.

O presente estudo examina a relação entre as TICs em meio a práticas colaborativas entre cliente-fornecedor e seu impacto no PDP. A Figura 3 apresenta o modelo que será utilizado neste trabalho.

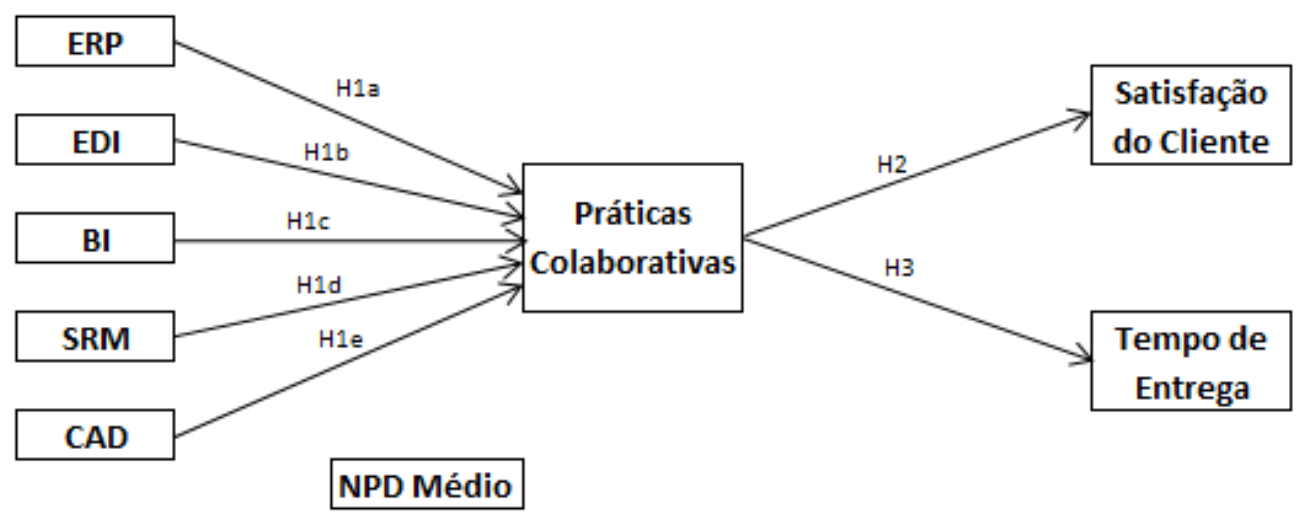

Figura 3. Modelo de Pesquisa

Fonte: Elaborado pelos autores (2016) 


\section{Procedimentos Metodológicos}

No trabalho foi realizada uma análise da utilização de ferramentas da tecnologia de informação que auxiliem na colaboração cliente-fornecedor no processo de desenvolvimento de produto. A partir disso, o estudo teve duas vias de análise principais: (i)A relação das ferramentas de TIC com as práticas colaborativas das empresas; (ii) Impactos das práticas colaborativas na performance dos produtos. A partir disso, pretende-se entender quais ferramentas de TIC tem um impacto positivo no desenvolvimento de produto colaborativo e também quais dimensões de desempenho do produto são beneficiadas com este processo.

O estudo se deu em empresas do Sul do Brasil, que utilizam ferramentas de TIC na gestão da cadeia de suprimentos, mais especificamente na relação com os fornecedores. Para isso, foram realizadas quatro etapas conforme a Figura 4.

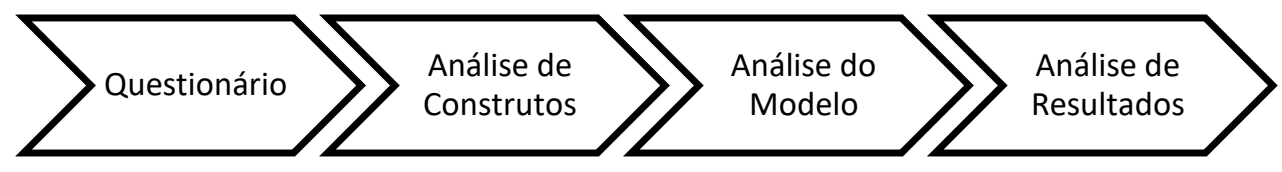

Figura 4. Etapas do trabalho

Fonte: Elaborado pelos autores (2016)

A primeira etapa foi feita através de um questionário que buscou diagnosticar as empresas analisadas quanto ao nível de utilização das TICs e foi aplicado para 240 participantes. Dos questionários retornados, 22 foram excluídos pois as empresas estavam na fase de planejamento da implementação e, 11 estavam ou incompletos ou não eram empresas localizadas no sul do Brasil, finalizando uma amostra de 110 respondentes. Os participantes responderam a cada questão da pesquisa utilizando uma escala Likert com cinco graduações de respostas. No caso da variável de níveis de utilização das TICs, 1 (um) correspondia a não utilizam e 5 (cinco) as utilizam totalmente e, 3 (três) as utilizava razoavelmente.

Para a seleção da amostra seguiram-se os seguintes critérios: (a) incluir empresas do Sul do Brasil, com o objetivo de eliminar os efeitos externos e garantir a homogeneidade dentro da amostra desses fatores, (b) incluir companhias de diferentes setores da indústria tendo em conta o crescimento da utilização de TICs e (c) escolher uma amostra de pessoas com experiência e conhecimento sobre o tema estudado. Os questionários foram aplicados em empresas pertencentes a diferentes setores como mostra a Tabela 1 .

Tabela 1. Composição da amostra

\begin{tabular}{lcc}
\hline Setor Industrial & & \\
\hline Automotivo & 27 & $24,5 \%$ \\
Alimentícia & 19 & $17,3 \%$ \\
Calçadista & 15 & $13,6 \%$ \\
Metalúrgica & 12 & $10,9 \%$ \\
Eletrônica & 11 & $10,0 \%$ \\
Tabagista & 8 & $7,3 \%$ \\
Bélico & 5 & $4,5 \%$ \\
Transporte e Logística & 4 & $3,6 \%$ \\
Petróleo e Energia & 3 & $2,7 \%$ \\
Fabricação de Móveis & 3 & $2,7 \%$ \\
Hidráulica & 3 & $2,7 \%$ \\
\hline
\end{tabular}

Fonte: Elaborado pelos autores (2016) 
Na segunda etapa foi feita a análises de construtos (Praticas colaborativas) no software Stata (Stata, Statacorp, College Station, TX, USA). E por fim foi feito o teste do modelo no software estatístico SPSS 19.0 (SPSS, IBM, Chicago, IL, USA) seguida da análise dos resultados obtidos.

\section{Análises e Resultados}

Após validar os dados, foi estabelecido um construto relativo a um conjunto de variáveis referentes às práticas colaborativas e verificado sua consistência através do software Stata. A Tabela 2 apresenta as variáveis que compõem o construto, assim como, os coeficientes que demonstram, ao apresentar valores maiores que 0,5 , que estas variáveis representam bem este construto. Já os valores encontrados dos indicadores CFI de 0,930 e TLI de 0,789 serviram para validar a confiabilidade do construto. Desta forma é viável que seja utilizado o construto no modelo de pesquisa, como representante das práticas colaborativas.

Tabela 2. Composição Construto

\begin{tabular}{lc}
\hline Construto [Práticas Colaborativas] & Coef $\odot$ \\
\hline Envolvimento de clientes do desenvolvimento de produtos & 0,705014 \\
Relações de longo prazo com fornecedores & 0,627210 \\
Informa frequentemente o desempenho aos fornecedores & 0,627210 \\
Entrega dos fornecedores por sistema puxado & 0,628373 \\
\hline
\end{tabular}

Fonte: Elaborado pelos autores (2016)

Em seguida a validação do construto, séries de múltiplas regressões lineares foram desempenhadas para testar as hipóteses apresentadas anteriormente.

Para testar nossas hipóteses H1a, H1b, H1c, H1d e H1e foi feita a regressão linear das TICs (ERP, EDI, BI, SRM, CAD) e NPD em relação às práticas colaborativas. Como pode ser observado na tabela 3, as tecnologias EDI e SRM estão fortemente associadas com o aumento de práticas colaborativas, validando H1b e H1d. A BI, por sua vez, apesar de não tão fortemente, também está positivamente associada com o aumento das práticas colaborativas, validando H1c. Já a ERP e CAD não apresentaram associação com o aumento das práticas colaborativas, invalidando H1a e H1e. Por fim, o NPD foi utilizado como variável de controle das hipóteses.

Agora para testar nossas hipóteses $\mathrm{H} 2$ e H3, foram feitas as regressões lineares das práticas colaborativas em relação à satisfação do cliente e ao tempo de entrega respectivamente. Como pode ser visto nas Tabelas 4 e 5, a adoção de práticas colaborativas está positivamente associada com o aumento da satisfação dos clientes e tempo de entrega. Porém, a associação de práticas colaborativas é mais forte com a satisfação do cliente do que com o tempo de entrega.

Tabela 3. Resultado de Regressão para Práticas Colaborativas

\begin{tabular}{llll}
\hline & \multicolumn{1}{c}{ Beta } & \multicolumn{1}{c}{$\mathbf{R}^{2}$} & Hipóteses \\
\hline VD: Práticas Colaborativas & & 0,504 & \\
NPD Médio & $0,296^{*}$ & & H1a \\
ERP & 0,085 & H1b \\
EDI & $0,268^{* * *}$ & H1c \\
BI & $0,126^{*}$ & H1d \\
SEM & $0,255^{* * *}$ & H1e \\
CAD & 0,970 & & \\
\hline
\end{tabular}

Obs.: VD = Variável

Dependente

$* \mathrm{p}<0,05, * * \mathrm{p}<0,01$ $* * * \mathrm{p}<0,001$

Tabela 4. Resultado de Regressão para Satisfação do Cliente 


\begin{tabular}{lccc}
\hline & Beta & $\mathbf{R}^{2}$ & Hipóteses \\
\hline $\begin{array}{l}\text { VD: Satisfação do Cliente } \\
\text { Práticas Colaborativas }\end{array}$ & $0,24 * *$ & 0,074 & $\mathrm{H} 2$ \\
\hline
\end{tabular}

Obs.: VD = Variável

Dependente

$* \mathrm{p}<0,05, * * \mathrm{p}<0,01$,

$* * * \mathrm{p}<0,001$

Tabela 5. Resultado de Regressão para Tempo de Entrega

\begin{tabular}{lccc}
\hline & Beta & $\mathbf{R}^{2}$ & Hipóteses \\
\hline $\begin{array}{l}\text { VD: Tempo de Entrega } \\
\text { Práticas Colaborativas }\end{array}$ & $0,134^{*}$ & 0,05 & H3 \\
\hline
\end{tabular}

Obs.: VD = Variável Dependente $\quad * \mathrm{p}<0,05, * * \mathrm{p}<0,01, * * * \mathrm{p}<0,001$

\section{Discussões e Implicações}

O uso da EDI representa mecanismos de comunicação rápida e aberta, o que é essencial para companhias que trabalham com atividades de SC, fazendo com que as empresas prevejam, desenvolvam, produzam, sincronizem e entreguem seus produtos/serviços antecipando-se as exigências dos clientes (MATTOS \& LAURINDO, 2015).

A BI tem um papel importante em produzir informações atualizadas para decisões operacionais e estratégicas. Através desta TIC o conhecimento e informações compreensivas e feitas a tempo colaboram para o NPD e melhoram as operações de negócios (HANNULA \& PIRTTIMAKI, 2003).

O SRM é o processo através de qual o negócio mantém o controle de seus fornecedores, usando um sistema de informações que automatiza o abastecimento, compra e administração da relação diária com o fornecedor (LEE et al., 2007). Esta tecnologia melhora o fluxo de demanda do produto e informações do fornecedor através da SC, levando a redução de custos e do tempo de entrega (CHOY et al.,2003).

Desta forma, trazendo de encontro à literatura com os resultados obtidos, podemos aferir que estas três tecnologias possuem papel importante na integração cliente-fornecedor para o PDP, trazendo resultados positivos para toda a cadeia.

Já os sistemas ERP são softwares que suportam grande parte das informações importantes da companhia entre as diversas áreas funcionais da empresa (KUMAR et al., 2000). A implementação do ERP colaborativo permite a coordenação e colaboração, entre várias empresas que geram valor para a rede, a automatizar o processo de fluxo de trabalho e o controle do fluxo de dados compartilhados entre as partes (ECKARTZ et al., 2009). Porém, segundo Eckartz et al. (2009) existem muito poucas pesquisas que tratam do ERP colaborativo. Isto dificulta a identificação, realização e avaliação dos benefícios desta TI na integração cliente-fornecedor. Alem disso, mais de 70\% das implementações de ERP falham em atingir os seus benefícios esperados (AL-MASHARI et al., 2006; STEFANOU, 2001). Assim, a falta de informações sobre o ERP colaborativo e as falhas de atingimento de objetivos da tecnologia demonstram algumas possibilidades para o resultado de H1a não ter sido favorável.

O CAD, por sua vez, é usado por engenheiros e especialistas de maneira interativa para projetar melhores produtos e para produzir com maior eficiência. Porém cada sistema de CAD tem a sua maneira específica de construir e modelar os dados geométricos. Como resultado, ao passar os dados de um sistema para outro, o sistema que recebe pode não conseguir manipular os dados (LICHTENBERG, 2007). Segundo Álvares \& Ferreira (2006) é improvável que todos os participantes do PDP usem os mesmos sistemas de hardware e software. Desta forma, é possível que o resultado desfavorável de H1e tenha ocorrido por este motivo.

Quando trata-se da satisfação do cliente e tempo de entrega, encontramos na literatura diversos autores que relacionam estes benefícios às praticas colaborativas. Segundo McLaren et al. (2002) o resultado do SCM colaborativo não é apenas a redução de perdas na SC, mas aumento de 
responsabilidade, satisfação do cliente e competitividade entre os membros da parceria. Para Akintoye et al. (2000), Matopoulos et al. (2007) e Sandberg (2007) diversos benefícios da colaboração vão sendo documentados durante os anos para produtores, fornecedores e clientes. Estes incluem redução de custos, melhora do serviço, melhora da satisfação dos clientes, redução do tempo de entrega, entre outros. A partir disso e dos resultados obtidos nas regressões lineares, podemos validar nossas hipóteses 3 e 4.

\section{Conclusões}

Muito se fala atualmente nas práticas colaborativas entre clientes e fornecedores em diversos aspectos, mas também em relação ao desenvolvimento de produtos. No meio disso, debate-se também a presença das TICs nesta relação. Este trabalho examinou, através de uma pesquisa realizada com 240 empresas da região Sul do Brasil, a utilização de ferramentas de TIC no PDP colaborativo e seu consequente impacto na performance da empresa. Desta forma, foi possível constatar, através do modelo apresentado, a existência de relação positiva entre algumas ferramentas de TIC com as práticas colaborativas entre as empresas, e também, a influência destas práticas na satisfação do cliente e no tempo de entrega do produto.

Em busca de continuar entendendo este processo e aprofundar os resultados obtidos até então, pretende-se realizar visitas formais às empresas para poder observar como são utilizadas na prática as ferramentas de tecnologia da informação para a colaboração, tanto nos clientes como nos fornecedores. Desta forma, pode-se ter uma percepção mais robusta da interação dos funcionários com as ferramentas de TIC e também uma visão mais ampla dos benefícios do uso destas tecnologias para as empresas envolvidas.

\section{Referências}

ABREU, Melissa C. O. Impacto da Tecnologia da Informação na Gestão da Cadeia de Suprimentos na Varig Engenharia e Manutenção S/A. 2006. 77 f. Dissertação (Trabalho de Conclusão de Curso em Administração) - Universidade federal do Rio Grande do Sul, Porto Alegre. 2006

AKINTOYE, A.; MCINTOSH, G.; FITZGERALD, E., A survey of supply chain collaboration and management in the UK construction industry, European Journal of Purchasing \& Supply Management, 6, 159-168, 2000.

AL-MASHARI, M.; ZAIRI, M.; OKAZAWA, K., Enterprise Resource Planning (ERP) Implementation: a Useful Road Map, Int. J. Management and Enterprise Development, pp 169180, 2006.

ÁLVARES, A. J.; FERREIRA, J. C. E., WebMachining: Implementation of a Collaborative CAD/CAPP/CAM System for EManufacturingThrough the Internet, Journal of Materials Processing Technology, 2006.

BANDEIRA, R. A. M; MAÇADA, A. C. G; Impacto da tecnologia da informação na gestão da cadeia de suprimentos do setor de gases industriais. XIV SIMPEP, 2007.

BARCZAK, Gloria; SULTAN, Fareena; HULTINK, Erik J. Determinants of IT usage and new product performance, The Journal of Product Innovation Management, vol. 24, pp. 600-613, 2007.

BORNIA, Antonio C.; LORANDI, Joisse A. O processo de desenvolvimento de produtos compartilhado na cadeia de suprimentos. Rev. FAE, Curitiba, v.11, n.2, p.35-50. 2008

CAMPOS, Lucas F. Integração dos Fornecedores no Desenvolvimento de Novos Produtos na Indústria Automobilística: Fatores Influenciantes e Critérios para Seleção de Parceiros. 2012. 
164 f. Tese (Graduação em Engenharia de Produção) - Escola Politécnica da Universidade de São Paulo, São Paulo. 2012.

CHOY, K.L.; LEE, W.B.; LO, V., Design of an intelligent supplier relationship management system: a hybrid case based neural network approach, Department of Industrial and Systems Engineering, The Hong Kong Polytechnic University, Hung Hom, Hong Kong, People’s Republic of China, 2003.

CHRISTOPHER, M. Logística e Gerenciamento da Cadeia de Suprimentos: Estratégias para a Redução de Custos e Melhoria dos Serviços. São Paulo: Pioneira Thomson Learnig, 2001.

ECKARTZ, Silja; DANEVA, Maya; WIERINGA, Roel; HILLEGERSBERG, Jos van, A Conceptual Framework for ERP Benefit Classification: A Literature Review, Information Systems and Change Management Group, University Twente, Enschede, the Netherlands, 2009.

FARIA, Adriana F.; PINTO, Ana C. A.; RIBEIRO, Maressa N.; CARDOSO, Tatiane S.; RIBEIRO, João P. C. Processo de Desenvolvimento de Novos Produtos: Uma Experiência Didática In: ENCONTRO NACIONAL DE ENGENHARIA DE PRODUÇÃO, 28, 2008, Rio de Janeiro. Anais... Rio de Janeiro: ABEPRO, 2008

FERRARI, Guilherme; POLACISKI, Édio. Gestão Estratégica em TI: uma abordagem conceitual de Supply Chain Management, Faculdade Horizontina, 2011.

FETTERMANN, Diego de C. Cooperação com Fornecedor no desenvolvimento de calçados no Rio Grande do Sul. 2008. 149 f. Dissertação (Mestrado em Engenharia de Produção) - Universidade federal do Rio Grande do Sul, Porto Alegre. 2008.

GASSMANN, O.; Enkel, E.Towards. A Theory of Open Innovation: Three Core Process Archetypes. Proceedings of the R\&D Management Conference, 2003.

HANDFIELD, R.B.; RAGATZ,G.L.; PETERSEN, K.J.; MONCZKA, R. M., Involving Suppliers in New Product Development.Calif.Manage.Rev.42(1),59-82, 1999.

HANNULA, Mika; PIRTTIMAKI, Virpi, Business intelligence empirical study on the top 50 Finnish companies, Journal of American Academy of Business, Cambridge; Mar 2003; 2, 2; ABI/INFORM Global pg. 593, 2003.

JOHNSON, Michael D.; FORNELL, Claes. "A Framework for Comparing Customer Satisfaction Across Individuals and Product Categories," Journal of Economic Psychology, 12 (2), 267-86, 1991.

KUMAR, K.; HILLEGERSBERG, J.V., Enterprise Resource Planning: Introduction, Communications of the ACM, pp 22-26, 2000.

LAWSON, Benn; PETERSEN, Kenneth J.; COUSINS, Paul D.; HANDFIELD, Robert B. Knowledge Sharing in Interorganizational Product Development Teams: The Effect of Formal and Informal Socialization Mechanisms, Journal of Product Innovation Management, 2009.

LE DAIN, M.A.;MERMINOD, V. A Knowledge Sharing Framework for Black, Grey and White Box Supplier Configurations in New Product Development: an exploratory analysis, Technovation. 7 October 2014.

LEE, Dongjoo; KANG, Seungseok; LEE, San-keun; KIM, Young-gon; LEE, Sang-goo, Best Choice SRM: A Simple and Practical Supplier Relationship Management System for e-Procurement, School of Computer Science and Engineering, Seoul National University, Seoul 151-742, Republic of Korea, 2007. 
LICHTENBERG, Joe, A New Approach to Eliminating Barriers to Collaboration in Multi-CAD Environments, Spaceclaim, 2007

MAÇADA, Antonio C. G.; FELDENS, Luis F.; SANTOS, André M. Impacto da tecnologia da informação na gestão das cadeias de suprimentos - um estudo de casos múltiplos. Gestão e Produção, São Carlos, v.14, n.1, p.1-12, jan.- abr. 2007.

MATOPOULOS, A.; VLACHOPOULOU, M.; MANTHOU, V.; MANOS, B., A conceptual framework for supply chain collaboration: Empirical evidence from the agri-food industry, Supply Chain Management: An International Journal, 12(3), 177-186, 2007.

MATTOS, Claudia A.; LAURINDO, Fernando J. B., Collaborative Platforms for Supply Chain Integration: Trajectory, Assimilation of Platforms and Results, Journal of technology Management \& Innovation, Volume 10, Issue 2, jun 18, 2015.

MCLAREN, Tim; HEAD, Milena; YUAN, Yufei, Supply chain collaboration alternatives: understanding the expected costs and benefits, Internet Research: Electronic Networking Applications and Policy, Volume 12, Number 4, 348 $\pm 364,2002$.

MUNDIN, Ana P. F.; ROZENFELD, Henrique; AMARAL, Daniel C.; SILVA, Sérgio L.; GUERRERO, Vander; HORTA, Lucas C. Aplicando o cenário de desenvolvimento de produtos em um caso prático de capacitação profissional. Gestão e Produção, São Carlos, v.9, n.1, p.1-16, abr. 2002.

OZER, M. 'Information technology and new product development: opportunities and pitfalls', Industrial Marketing Management, Vol. 29, pp.387-396. 2000.

PETERSEN, K. J.; HANDFIELD, R.B.; RAGATZ,G.L.,2005.Supplierintegrationintonew product development: coordinating product, process and supply chain design. J. Oper. Manage. 23(1), 371388. 2005.

PORTER, Michael. Vantagem competitiva: criando e sustentando um desempenho superior. 15. ed. Rio de Janeiro: Campus, 1989.

RAGATZ, G.L.; HANDFIELD, R.B.; SCANNELL, T.V.Success Factors for Integrating Suppliers into New Product Development. Journal of Product Innovation Management 14(3):190-202. 1997.

ROZENFELD, H.; FORCELLINI, F.A.; AMARAL, D.C.; TOLEDO, J.C.; SILVA, S.L.;

ALLIPRANDINI, D.H.; SCALICE, R.K. Gestão de Desenvolvimento de Produtos: uma referência para a melhoria do processo. São Paulo: Saraiva, 2006.

SANDBERG, E., Logistics collaboration in supply chains: Practice vs. theory, The International Journal of Logistics Management, 18(2), 274-293, 2007.

SANTOS, Fabio L.; MACHADO, João H.; NANTES, José F. D. Gestão da Parceria ClienteFornecedor no Processo de Desenvolvimento de Produtos In: ENCONTRO NACIONAL DE ENGENHARIA DE PRODUÇÃO, 30, 2010, São Carlos. Anais... São Carlos: ABEPRO, 2010.

SILVA, Chathurani; MATHRANI, Sanjay; JAYAMAHA, Nihal. A framework to evaluate the impact of ICT usage on collaborative product development performance.4th Annual New Zealand Information Systems Doctoral Consortium, 2013.

STEFANOU, C.J., A Framework for the Ex-ante Evaluation of ERP Software, European Journal of Information Systems, pp 204-215, 2001. 
YI, Youjae, “A Critical Review of Consumer Satisfaction," in Review of Marketing 1990, ed. Valarie A. Zeithmal, Chicago: American Marketing Association, 68-123. 1991. 